\title{
La linguistique française et son histoire
}

\author{
Sylvain Auroux \\ Laboratoire d'histoire des théories linguistiques, UMR 7597
}

\section{Un domaine actif des sciences du langage}

Parmi les innovations les plus importantes des sciences du langage durant ces trente dernières années, il faut incontestablement ranger le développement de leur histoire. Nous disposons désormais de nouvelles synthèses ${ }^{1}$, d'innombrables monographies et d'importantes discussions qui se déroulent lors de rencontres régulières. Le mythe d'une naissance récente des sciences du langage (apparu au $19^{\text {ème }}$ siècle) n'est plus guère tenable. Nous savons les faire remonter aux paradigmes que l'on trouve dans les marges de bilingues sumérien/akkadien au tournant des $3^{\text {ème }}$ et second millénaires avant notre ère. Comme toute discipline scientifique l'histoire des sciences suppose des résultats. Il peut s'agir de descriptions conceptuelles, de la construction de modèles d'évolution ou de l'évaluation de la place d'un thème ou d'un auteur dans un champ théorique. Les communications rassemblées dans la session « Histoire et Epistémologie » du CMLF 2008 appartiennent à toutes ces catégories. Il est difficile de dire que l'histoire de la linguistique française n'est pas bien documentée, puisque l'histoire de la grammaire française remonte au $18^{\text {eme }}$ siècle et que la quantité de documents rassemblés, par exemple, dans l'Histoire de la langue française de F. Brunot demeure encore largement inexplorée, sans parler de ceux rassemblés dans les différents répertoires publiés par A. Chervel. Pourtant, s'agissant de l'histoire de la linguistique française (et même des sciences du langage en général) nous ne sommes guère dans la situation des physiciens ou des mathématiciens. Dans ces disciplines : i) chaque chercheur a consciences de travailler à accroître des connaissances; ii) il y a un consensus pour définir les grandes étapes et les grandes inventions. Je ne connais pas un linguiste qui sache dater et citer dix grandes inventions en matière de théorie linguistique en recueillant l'accord de ses collègues. C'est également vrai pour la linguistique française.

La question est plus complexe qu'elle y paraît. Supposons que je demande: «qui a inventé la classification du conditionnel français, non plus comme un mode, mais comme un temps? ». Le jeune linguiste ignorera probablement la réponse, voire la pertinence de la question; le linguiste plus âgé ${ }^{2}$ renverra à Temps et verbe de G. Guillaume (1929), où le «psychomécanicien » le décrit comme un futur hypothétique; s'il lit le texte (on ne voit pas pourquoi !), il remarquera que Guillaume critique quelques auteurs qui en ont fait un « imparfait du futur, tout en maintenant l'existence du conditionnel mode » et là, on se demande de qui Guillaume parle. L'historien de la linguistique bien informé, citera l'idéologue Destutt de Tracy, qui dans sa Grammaire (1803) décrit les conditionnels français comme des « imparfaits des temps à venir ». Mais on ne sait toujours pas qui sont les grammairiens visés par Guillaume (Destutt ne conserve pas le conditionnel comme mode). Dans le fond, le problème n'est pas là. Il est dans le fait que le linguiste ne verra dans cette discussion qu'un point d'érudition. Dans son langage, il est prêt à admettre que Guillaume est l'inventeur de la psychomécanique; à l'inverse, je ne connais, dans la littérature, aucune trace d'une expression, comme l'invention de l'imparfait ${ }^{3}$. Autrement dit, nous admettons facilement qu'il y a invention (ou création) d'un système, rarement qu'il y a invention (découverte) d'un phénomène. C'est ce qui distingue essentiellement la linguistique et, disons, la physique, dans leur rapport à leur histoire. On pourrait en tirer trois hypothèses quant à la nature des sciences humaines, et, particulièrement, des sciences du langage :

(i) il n'y a pas d'invention dans ce domaine, mais des systèmes de représentations entre lesquels le choix est plus ou moins arbitraire (question d'époque ou de goût);

(ii) il n'y a pas d'invention dans ce domaine, parce que ces disciplines ne bénéficient pas de la même maturité que les sciences de la nature (on le dit trivialement : «ce ne sont pas encore des sciences »); 
(iii) il n'y a pas d'invention dans ce domaine, parce qu'une « invention » suppose qu'on puisse isoler un élément transcendant les théories («la loi de Mariotte»; « la loi des aires »), tandis que les éléments n'ont de sens qu'au sein d'un système (holisme).

Ces hypothèses présupposent toutes l'idée qu'il n'y a pas de «cumulation » dans le domaine; manifestement, aucune n'est satisfaisante. D'abord, parce que l'ancienneté de la grammaire rend extrêmement difficile que l'on admette la typicalité de la physique mathématique pour le concept de science. Ensuite, parce qu'un historien un peu attentif est capable, dans certains cas de retracer sur le long terme l'histoire de certains éléments qu'on peut appeler des « inventions » (par exemple, faire le rapport entre la théorie des synonymes et la conception saussurienne de la valeur). Enfin, parce que nous connaissons, dans certains domaines des sciences du langage, une cumulation des résultats, analogue à ce que l'on trouve ailleurs. C'était le cas, chez les générativistes, pendant une vingtaine d'années, lorsqu'on répertoriait les principes («Principe A sur A »), en les nommant parfois du nom de leur « inventeur» (contrainte de Emonds »). Le domaine le plus flagrant est sans doute la grammaire comparée indoeuropéenne : dès le $19^{\text {ème }}$ siècle on répertorie les « lois » (loi de Grimm, de Grassmann, de Verner, etc.), généralement sous le nom de leur inventeur ${ }^{4}$, pratique qui se poursuit jusqu'à nos jours comme en témoigne la publication de compendia ${ }^{5}$ (voir, par exemple, N. E. Collinge, The Laws of Indo-European, Amsterdam, John Benjamins, 1985). Dès lors, on doit présenter deux nouvelles hypothèses :

(iv) La cumulation est possible dans certains domaines des sciences du langage qui ont atteint leur maturité scientifique ; il s'agit d'une nouvelle version de (ii);

(v) La cumulation dépend d'un dispositif social qui fixe des finalités, trie et organise une « mémoire »; ce dispositif n'existe pas au même degré dans les sciences de la matière et les sciences humaines (resterait à expliquer pourquoi).

On se convaincra vite que ces deux hypothèses ne sont guère exclusives. La dernière, si elle présente une explication, ne fournit guère de solution immédiate : à celui qui soutiendra qu'il suffit d'organiser la cumulation, on fera remarquer que celle-ci dépend d'un consensus et que c'est justement ce dernier qui fait défaut : comment le faire exister?

On aurait probablement tort de soutenir une équation du genre :

(vi) science $=$ cumulation $=(\mathrm{v})$

Dans le fond, le mode de cumulation moderne de la science ne s'est guère développé avant le $19^{\text {ème }}$ siècle et, il est sans doute abusif de confondre l'existence de la science avec un mode particulier d'organisation sociale. L'historien soutiendra, en effet, qu'avec ou sans dispositif social de cumulation explicitement construit, avec ou sans vision historique des résultats acquis, il se publie des grammaires, et celles-ci ne sont pas indépendantes les unes des autres. Du seul fait qu'il y a de la temporalité, il se passe quelque chose, bref, en tout état de cause, il y a une « histoire ». Or, il nous faut avoir une représentation « de ce qui se passe » pour comprendre justement en quoi consiste cette « histoire » et éventuellement voir quel intérêt il y aurait à vouloir changer les choses. J'ai le sentiment que ce colloque a apporté quelques éléments nouveaux dans cette direction.

\section{L'histoire sérielle}

Soient les définitions suivantes d'une «catégorie grammaticale », la préposition; si l'on considère la première avec les autres, on est dans le long terme (15 siècles), la seconde et les deux autres dans le moyen terme (un siècle), les deux dernières dans le court terme (dix ans).

La préposition est un mot qui se prépose à toutes les parties de phrase en composition et en construction (Denys le Thrace, III $^{\circ}-\mathrm{IV}^{\circ}$ siècle)

Ce sont presque les mêmes rapports dans toutes les langues qui sont marqués par les prépositions (Port-Royal, 1660) 


\begin{abstract}
Des mots qui expriment des rapports généraux, avec indétermination de tout terme antécédent ou conséquent (Beauzée, 1767)

Il y a des prépositions qui, en indiquant le second terme d'un rapport, expriment encore le rapport même, et qui, par conséquent, modifient le premier terme (Condillac, 1775)
\end{abstract}

On peut d'emblée se demander s'il y a un sens à aligner ces définitions. Certes, nous avons une identité de terminologie (modulo une traduction), mais il est difficile de voir un rapport immédiat entre la première définition et la suivante. Visiblement entre la première et la seconde, on a franchi une barrière épistémologique : on est passé d'une définition purement "formelle » à une définition sémantique. Qu'est-ce qui nous assure que, d'une façon ou d'une autre, on parle bien de la " même » chose, puisqu'il n'y a aucune intersection entre les définitions? Aucune grammaire ne fonctionne sans donner des exemples; de Denys à Condillac, nous sommes capables de voir que la catégorie subsume plus ou moins le même type de mots, ou, disons, des mots qui ont un " air de famille ». Un concept n'est pas une unité sémantique donnée une fois pour toutes, ou renouvelé de fond en comble à chaque définition, à chaque insertion dans un complexe théorique différent. C'est un complexe composé d'un vocabulaire, d'autres concepts, de définitions, d'exemples canoniques, de textes de références, de problèmes distingués. Tous ces éléments peuvent changer; la stabilité tient seulement au fait que tous ne changent pas en même temps.

Quand il y a changement sur une série donnée, on peut se demander : si ce changement est orienté ou si les éléments postérieurs ont plus de «valeur» (par exemple, sont empiriquement plus adéquats) que les antérieurs; éventuellement, on peut aussi se demander, si ces derniers ont quelque influence sur la formations des premiers. Il s'agit de questions peu claires, dont l'obscurité est souvent dissimulée sous le concept uniformisant de «progrès » ou sous celui de « rupture », construit de façon ad hoc pour pallier les défauts du premier. Entre le premier concept et le second, on a vu la rupture ; d'une certaine façon, aucune des deux définitions pourtant n'annule l'autre et il y a également «cumulation », dès qu'on les « additionne ». Il en irait de même si j'ajoutais à la série des éléments qui proviennent de langues où les « prépositions » seraient postposées (toutefois, le centre de la définition bascule alors sur la sémantique et il faut restreindre à certaines langues la portée de celle de Denys). Ou si après avoir exhibé des éléments dans lesquels on insiste sur le caractère invariable des prépositions, j'en ajoute qui font état de langues où elles se conjuguent. On pourrait imaginer une rupture telle que des éléments définitoires soient définitivement inconsistants, quelle que soit l'habilité mise à chercher une compatibilité (par exemple, comme on vient de le faire en changeant les langues de référence). Pourrait-on avoir des éléments définitoires totalement incompatibles, en gardant des ensembles d'exemples canoniques non disjoints ? J'avoue être incapable de me représenter ce que cela pourrait signifier. A l'inverse, on remarque que la grammaire dispose, avec les catégories grammaticales, de «noyaux de rationalité » relativement stables depuis l'Antiquité.

La question de la «causalité » est relativement complexe et diversifiée. D’abord, il faut que l' «antérieur» figure dans la connaissance de ceux qui élaborent le "postérieur», soit sous forme explicite, soit sous la forme anonyme de la «connaissance commune ». On peut être assuré de la permanence du concept de Denys; au reste, il suffirait d'ajouter des éléments à notre série pour le montrer avec plus de précision. Sur le très long terme, cela n'apporte pas grand-chose, sauf à dire «c'est $\mathrm{X}$ qui a inventé (découvert) le concept $\mathrm{C}_{\mathrm{i}}$ » (pour la préposition, ce seraient les philosophes stoïciens, plutôt que le grammairien Denys). Sur le moyen terme, Beauzée connaissait évidement Port-Royal, dont on peut dire qu'il reprend la définition en la précisant : une préposition peut recevoir différents arguments (la généralisation à «n'importe lequel », au reste, serait discutable : on peut dire " après demain », mais pas « après hier »). Dans le court terme, il faut des analyses plus fines. "Indiquer » est un concept clé de la sémiotique de Condillac; il s'agit d'un mode de référence indirect qui ne nécessite pas de représentation de l'objet (on peut nommer un entier, on ne peut qu'indiquer un réel, parce que ce dernier suppose une suite infinie). On voit bien qu'il y a une relation avec l'indétermination de Beauzée: l'absence de signification assignée. La préposition est le premier élément linguistique sur lequel ce phénomène semble avoir été mis en lumière. 
L'absence du dispositif social moderne de cumulation des résultats n'empêche ni une relative conservation sur le long terme, ni l'existence, sur le court terme, d'une « orientation » assez claire du changement. Tout simplement parce qu'il n'existe pas de «connaissance instantanée » : il faut du temps pour savoir et un individu n'y suffit pas. On pourrait opposer le dispositif social moderne à tout le reste, que l'on pourrait baptiser «cumulation sauvage ». En quelque sorte, il s'agit d'une question de degré de liberté (de quantité de « libéralisme ») dans la production scientifique : pour différentes raisons, qui ne nous concernent pas ici, les sciences modernes comme la physique ou la biologie, sont très « encadrées ». Je ne suis pas sûr toutefois que l'on puisse parler absolument de «cumulation sauvage ». D'abord, il est probable que cette catégorie recouvre une grande diversité. Ensuite, je ne pense pas qu'il puisse y avoir «cumulation » en l'absence de toute régulation, fût-elle implicite. La nécessité de se référer à quelques grands textes est une régulation minimale qui possède à l'évidence un effet cumulatif, pas toujours bénéfique d'un point de vue historique : elle conduit à surévaluer certains auteurs et, probablement, à imaginer, quand la série s'allonge, sans qu'il y ait suffisamment d'intermédiaires, des ruptures artificielles.

Dans le domaine des sciences du langage en général et de la grammaire française en particulier, l'historien ne peut s'appuyer sur un concept unifié (encadré) de la discipline. Méthodologiquement, il ne lui reste qu'à privilégier une démarche « empirique ». La solution c'est l' « histoire sérielle »: il s'agit de construire un corpus représentatif et de suivre la constitution et/ou les variations d'un «concept» ( « proposition », « adjectif », « verbe », « temps », « complément », « détermination », etc.). C'est le « concept» qu'il faut suivre dans un corpus important. On imagine le travail de dépouillement, ingrat, mais indispensable à l'historien. Une histoire de la grammaire française construite sur ces prémisses est en train de naître ; en témoignent plusieurs exposés de ce colloque.

Un corpus représentatif est une série choisie, dont l'élaboration est déjà un travail théorique (des indicateurs externes comme le taux de réédition sont certes importants mais insuffisants), sur lequel nous ne pouvons guère nous attarder. Je voudrais seulement mettre en lumière un autre problème. On peut le formuler brutalement de la façon suivante : un concept (au sens où nous l'avons défini), apparaît-il lorsque se stabilise un nom pour le manier?

J.-C. Chevalier, il y a exactement quarante ans, dans une thèse brillante et fondatrice, étudiait l'apparition de la notion de complément. Il arrêtait son Histoire de la notion de complément (Droz, 1968), à l'apparition du mot chez Beauzée (où il ne s'agit que d'un élément assez éloigné du phénomène de transitivité), soit vers le milieu du $18^{\text {ème }}$ siècle; il ne disposait guère d'information sur l'histoire de la logique et de la notion de proposition, ni d'instruments méthodologiques très performants ; enfin, aucune étude sur des concepts adjacents (proposition, verbe, détermination, temps, adjectifs, etc.) n'était encore disponible. Une thèse récente (2007) - dont on trouvera l'écho dans l'une des communications au colloque - reprend la question sous le titre «régime, complément et transitivité 1651-1863 ». Elle aborde, au départ le très long terme (depuis l'Antiquité) et traite la question en dehors de la problématique naïve de la présence (invention)/ absence d'un «concept» scientifique (qui au reste ne serait guère défini). C'est pourquoi trois noms de «concepts» figurent dans son titre. Il s'agit d'un changement profond pour la représentation de l'histoire d'une science. On peut admettre qu'il arrive que certains phénomènes ne soient tout simplement pas représentés/représentables dans un état donné d'une discipline. C'est rarement le cas pour des phénomènes massifs. On assiste alors à différentes représentations, à chaque fois avec "les moyens du bord». Il est ainsi difficile de dire que les grammairiens de l'Antiquité «ignoraient» le phénomène que nous abordons depuis le milieu du $19^{\text {ème }}$ siècle sous le nom de complément. Ils le traitaient (avec plus ou moins de bonheur), par exemple, en mettant au point une classification des différents types de verbes ou en abordant la transformation passive des verbes qu'ils classaient comme «actifs ». C'est un phénomène assez général dans l'histoire de la grammaire. Dès lors, il faut lui donner un statut. Cela revient à comprendre les motivations du changement.

Pour cela, il faut suivre la thématisation du phénomène qui nous intéresse et pointer dès le départ quelques éléments qui vont avoir un poids théorique considérable. Le premier est l'origine absolument logique de la notion de proposition (Aristote) ; le second est le fait (contingent, donc) que la structure propositionnelle prédicative $(S$ est $P$ ) mise au jour par Aristote sera prédominante en Occident (alors que 
d'autres structures, par exemple, celle que proposaient les stoïciens, étaient disponibles); le troisième est le fait que la grammaire, qui développe ses instruments propres (cas, régime, transitivité, ce dernier reposant sur un modèle physique de ce que représente l'énoncé), n'utilise que tardivement la notion de proposition. On sait que le modèle aristotélicien de la proposition a lourdement obéré le développement de la logique parce qu'il interdit toute conception des relations (et donc du discours mathématique). Dans ces conditions, l'importation du modèle en grammaire - largement dû à Port-Royal - ouvre, symétriquement, pour les linguistes, la question du complément : dans " (Socrate) (est mortel)», le modèle propositionnel Sujet-Prédicat, fonctionne parfaitement; dans « (Socrate) (aime la sagesse) », il conduit à négliger ce que peut-être la fonction de «sagesse ». Pour en construire la représentation, il faudra «casser» la notion de «prédicat». Cela correspond moins à la découverte d'un nouveau phénomène qu'à la résolution d'un problème que l'on peut formuler ainsi : comment intégrer dans la structure propositionnelle qui sert désormais à analyser la phrase, la représentation des phénomènes abordés par des concepts comme «régime» («syntaxe de régime ») ou «transitivité »? Ce que nous montre l'histoire sérielle est une situation complexe à laquelle la notion de «progrès» et donc de « seuils» de découvertes (en l'occurrence, la notion de «complément ») n'est guère appropriée. D'un côté, nous avons bien une certaine «identité » conceptuelle sur le long terme, assurée par l'identification des phénomènes et la persistance de corpus canoniques. De l'autre nous avons une ré-analyse totale. En quoi pouvons-nous dire que cette analyse est «meilleure», qu'elle représente un «progrès », comme lorsque l'on dit «Beauzée a découvert la notion de complément» (sous-entendu : avant on en ignorait tout). On comprend que j'ai choisi un exemple éloigné dans le temps, mais que la question vaut pour tout modèle contemporain. Ce n'est pas que je sois absolument pessimiste : je ne vois pas comment on pourrait continuer à avancer, en se contentant de quelque forme de cumulation sauvage que ce soit, laissant place aux aléas de l'oubli incontrôlé et à la tentation illusoire de croire que tout recommence à chaque prise de parole socialement un peu forte.

\section{Le paradoxe historiographique}

Dans le domaine de la grammaire, il faut donc se résoudre à ce que l'historien procède par l'étude empirique, avec ses propres méthodes (histoire sérielle), sans l'aide d'une véritable conception « encadrée » de la discipline. On pourrait imaginer que les résultats du travail historique ait un impact direct sur la pratique de la grammaire. En fait, la situation est beaucoup plus complexe. D'abord, dans le contexte actuel, il n'y a aucune raison pour que les linguistes, qui ne se lisent guère d'une école à l'autre, fassent véritablement l'effort d'une culture historique indispensable à ce que les choses changent. Ensuite, il faut compter avec le «paradoxe historiographique $»^{6}$.

L'ordre argumentatif du discours scientifique ou, par facilité, disons l'ordre logique de l'agencement du raisonnement, est totalement étranger à l'indexation temporelle des propositions. Plus simplement, on peut dire que l'introduction d'une telle indexation (par exemple : «Chacun sait que X a inventé Y dans son célèbre ouvrage $Z$ de $1957 »)$, a pour effet de transformer en incise la proposition qui en est affectée ; autrement dit, sa valeur de vérité ne joue aucun rôle dans celle de l'argumentation. La conséquence directe est claire : dans le discours scientifique, la valeur de vérité des remarques historiques qu'on peut y trouver n'entrent pas en ligne de compte. Autrement dit, on peut dire à peu près n'importe quoi, ça ne compte pas! On rejoint le sentiment de certains scientifiques qui voient dans les recherches historiques une activité anecdotique de pure érudition, sans impact réel.

Cette attitude serait parfaitement justifiée comme conséquence du paradoxe historiographique, si une science était un pur système de propositions. Or, il n'en est rien ${ }^{7}$. En particulier, on peut admettre facilement dans le cas de la science, la thèse de Putnam selon laquelle il n'y a pas de démarcation claire entre les faits (la science) et les valeurs ${ }^{8}$. C'est pourtant une règle absolue (je veux dire incontestée) de la méthode scientifique que l'on ne doit pas introduire de jugement de valeur dans l'argumentation scientifique. On ne doit pas même introduire la règle que l'on vient de citer, sous peine de la contredire. Dans ces conditions comment intervient-elle ? Elle fait partie des «contraintes » -- que chacun apprend plus ou moins implicitement durant sa formation -- qui pèsent sur l'élaboration du discours scientifique 
sans que ces contraintes puissent être inscrites au sein de ce discours lui-même. Cela ne veut pas dire qu'avec ou sans cette « contrainte », le discours sera le même. Autrement dit, le système de valeur (ou les différents systèmes possibles) qui président à l'élaboration du discours scientifique est (sont) fondamental pour la valeur de ce discours lui-même. C'est un statut de ce type qu'il faut revendiquer pour l'histoire des sciences. Une proposition historique (indexée temporellement) a une valeur de vérité et le plus souvent elle s'exprime également sur la valeur de vérité d'une connaissance. Un exemple montrera mieux l'enjeu de la question.

On connaît le mythe vivace ${ }^{9}$ d'un Jones fondateur du comparatisme, parce qu'il aurait « découvert » le sanskrit ${ }^{10}$. La comparaison des langues n'est pas une nouveauté lorsque Jones travaille. Il appartient même à un contexte général de compilations sur la diversité des langues consécutive au développement des échanges et à l'expansion occidentale, qui a produit pour les langues du monde, la révolution technologique de la grammatisation. Dès que l'on dispose de suffisamment d'informations sur un groupe de langues, on propose les apparentements sur la base des ressemblances intuitives: $17^{\text {ème }}$ siècle, langues sémitiques et néo-latines $; 18^{\text {ème }}$ siècle, familles caribe, algonkine, sioux, austronésienne (ou plutôt, comme on dira au $19^{\text {ème }}$ siècle «malayo-polynésienne »), finno-ougrienne. Le succès est moins évident sur les langues indo-européennes, avant l'intégration approfondie du sanskrit, dont les premiers éléments sont connus dès la Renaissance, et dont la première grammaire occidentale paraît en 1790. On ne le dira jamais asse : à la fin du $18^{\text {ème }}$ siècle, les linguistes disposent de méthodes déjà largement codifiées (depuis la Ranaissance) pour comparer les langues. De manière générale, quand les langues sont bien connues, ils mettent en parallèle des dictionnaires et des éléments grammaticaux (voir, par exemple, Gyarmathi sur les langues finno-ougriennes); quand elles le sont moins (voire très peu), ils utilisent soit des tableaux de mots élémentaires, soit la traduction d'un texte court (généralement le Notre père). Si cette méthode permet d'éclatants succès, lorsque l'on est effectivement à l'intérieur d'un groupe de langues apparentées, elle mène à des catastrophes lorsqu'on l'étend sans restriction. C'est précisément ce qui se passe lorsqu'on l'utilise lors d'une recherche de la parenté entre toutes les langues pour soutenir le monogénétisme. Le programme de Court de Gébelin en est un bon exemple. Le représentant des Eglises Réformées de France a dilapidé toute sa fortune pour construire un Dictionnaire de la langue primitive. Le problème est que sa méthode de comparaison multilatérale conduit à obscurcir les apparentements : dans ce contexte, tout rapprochement est irréfutable, tout ressemble nécessairement à tout. Il faudra une quarantaine d'années pour réfuter son programme et mettre en lumière l'insuffisance de la méthode multilatérale $e^{11}$

Où situer W. Jones ? C'est incontestablement un bon grammairien (on lui doit notamment une grammaire du persan) et son rôle a été considérable pour le développement des études concernant la langue et les textes sanskrits, quand bien même il est totalement erroné de soutenir qu'il aurait « découvert»le sanskrit. Cependant, comme comparatiste, il est dans la droite ligne de la science de son temps et des méthodes à la Court de Gébelin. Sa célèbre adresse de 1786 à l'Asiatic Society de Calcutta (celle où de nombreux auteurs imaginent encore pouvoir trouver l'origine de la grammaire comparée) le prouve amplement. Certes le juge de Calcutta fait bien l'hypothèse que les affinités entre le grec, le persan et le sanskrit témoignent d'une origine commune (l.c., p. 15). Mais dans le même texte ${ }^{12}$, il note que les habitants de l'Inde ont une «immemorial affinity» avec les "anciens Perses, Ethiopiens, Egyptiens, Phéniciens, Celtes, Chinois, Japonais et Péruviens », et comme ces nations ne sont pas des colonies les unes des autres, il en conclut que « toutes sont venues de quelque contrée centrale » (l.c., p. 20). Comme Court, Jones ne sait pas arrêter la comparaison et milite pour un monogénétisme sans garantie scientifique.

La conclusion qui s'impose à l'historien des sciences c'est que, contrairement à la légende, la grammaire comparée n'est pas née brutalement de la «découverte » par Jones du sanskrit. La pratique de la comparaison des langues et la recherche de leurs apparentements fait partie de la «science normale » depuis la Renaissance. Elle produit d'importants résultats et connaît une forte croissance dans le dernier tiers du $18^{\text {ème }}$ siècle. Le comparatisme moderne pourtant est l'effet, non d'une pseudo découverte empirique et ponctuelle du sanskrit, mais d'une révolution épistémologique, due à Rask, Grimm ${ }^{13}$ et Bopp. Il s'agit d'un changement de programme scientifique, qui a lieu dans le contexte d'une «science 
normale », déjà fortement densifiée. Quand cette révolution se sera stabilisée, au milieu du $19^{\text {ème }}$ siècle, l'unité de référence ne sera plus le mot mais le son, qui change dans toutes ses occurrences en fonction de lois phonétiques spécifiques à une époque et pour des langues données.

Le «mythe W. Jones » n'est pourtant pas sans conséquences. Il accrédite l'idée que la « révolution comparatiste » dépend de connaissances purement factuelles et de comparaisons multilatérales; il situe cette révolution dans des méthodologies qui ne sont pas les siennes et qui sont enracinées dans une recherche sur l'origine des langues, que justement le comparatisme refusera. Une science animée par le «mythe W. Jones » ne pouvait guère éviter la catastrophe intellectuelle que représente les recherches de M. Ruhlen sur l'origine des langues.

Evidemment, il n'existe peut-être pas d'exemple aussi caricatural dans le domaine de l'histoire de la grammaire française. Néanmoins nous pouvons être assurés que le travail historique est une nécessité scientifique.

${ }^{1}$ S. Auroux (dir.), Histoire des idées linguistiques, 3 vols, Liège, Mardaga, 1989-2000 ; G. C. Lepschy (dir.), Storia della linguistica, 3 vols, Bologne, Il Mulino, 1990.; History of the Language Sciences/ Geschichte der Sprachwissenschaften/ Histoire des sciences du langage, S. Auroux, E.F.K. Koerner, H.-J. Niederehe \& K. Versteegh (eds), Berlin \& New York, Walter de Gruytet, 3 vols, 2000-2005.

${ }^{2}$ On sait le rôle qu'ont longtemps joué les guillaumiens dans la préparation à l'agrégation des Lettres.

${ }^{3}$ Evidemment, je ne me compte pas dans le lot : j'ai coutume de dire que l'invention de l'imparfait, qui suppose un double repérage (antériorité par rapport au moment de l'énonciation et simultanéité par rapport à un autre événement) remonte au grammairien byzantin médiéval, Maxime Planudes.

${ }^{4}$ Sauf quand plusieurs auteurs peuvent en revendiquer la paternité, ce qui rend également difficile la datation (« loi des palatales »).

${ }^{5}$ Cela n'implique pas que l'historien y trouve automatiquement son compte: dans l'ouvrage cité, Collinge répertorie (p. 248) sous le nom de Kronasser à la date de 1951, une loi selon laquelle la signification des mots évoluerait du concret vers l'abstrait. Depuis le $17^{\text {ème }}$ siècle au moins, il s'agit d'un des lieux communs de la philosophie empiriste !

${ }^{6}$ S. Auroux, “L’histoire des sciences et le paradoxe historiographique”, Le Gré des langues n 8, 1995, 45-63.

${ }^{7}$ Voir, dans S. Auroux, 2007, La question de l'origine des langues suivi de L'historicité des sciences, Paris, PUF, la seconde partie.

${ }^{8}$ H. Putnam, Fait/Valeur: la fin d'un dogme et autres essais, Editions de l'Eclat, Paris, Tel-Aviv, 2004 (or. Angl. 2002).

${ }^{9}$ Sur toutes ces questions qui sont pourtant bien documentées voir, par exemple, le tome 2 de l'Histoire des idées linguistiques, 1992, Liège, Mardaga, en particulier, 538-579.

${ }^{10}$ Voir, par exemple, M. Ruhlen, 1994, The origin of Language. Tracing the Evolution of the Mother Tongue, New York, John Wiley \& Sons, Inc., p. 26-28 ou, encore, B. Laks, «Le comparatisme : de la généalogie à la génétique », Langages $\mathrm{n}^{\circ} 126,2002$, p. 19 : «Il est difficile d'imaginer aujourd'hui l'effet révolutionnaire qu'eut en son temps la proposition de Jones dans le monde de l'érudition ».

${ }^{11}$ Et plus encore pour rendre officielle l'idée qu'on ne peut atteindre l'origine des langues par des moyens linguistiques.

${ }^{12}$ Il s'agit de la troisième adresse (1786) à l'Asiatic Society pour l'anniversaire de sa fondation et publiée dans son célèbre journal. On la trouve reproduite dans W. Lehmann, A Reader in Nineteenth-Century Historical IndoEuropean Linguistics, Indiana University Press, Bloomington et Londres, 1967, p. 7-20; c'est le texte que nous citons. Il y a déjà plus de vingt ans que G. Metcalf (1984, "Adelung discovers the language of Asia", HEL VI-2, 101-115 p. 110) a attiré l'attention sur le passage auquel nous nous référons et qui constitue la conclusion du texte.

${ }^{13}$ La seconde édition de la Vergleichende Grammatik der deutsche Sprache [...], 1822, formule la célèbre «Lautverschiebung », la mutation consonantique qui marque la séparation des langues germaniques au sein des langues indo-européennes; il s'agit de la première formulation moderne d'une " loi phonétique » (basée sur le son et non le mot et régulière dans son fonctionnement), même si sa présentation (sous forme de cycles) est trompeuse et 
ISBN 978-2-7598-0358-3, Paris, 2008, Institut de Linguistique Française

Histoire, épistémologie, réflexivité

DOI $10.1051 / \mathrm{cmlf0} 8337$

largement erronée. Une grande partie des recherches de la seconde moitié du siècle visera à trouver (avec succès) une explication pour chaque exception rencontrée aux « lois de Grimm ». 